\title{
FUZZY DECISION ON TRAVERSE SPEED FOR ABRASIVE SUSPENSION JET
}

\author{
Wenbin Wang, Tao Yu, Xiaojian Liu, Tan Liu \\ CIMS \& Robot Center, Shanghai University, Shanghai, PR China,Email: \\ wenbin_wang@126.com
}

Abstract: The traverse speed is very important parameters of cutting process, But the cutting process of the ASJ (abrasive suspension jet) is a complex system with many interactions and influences among process parameters. It is hard to value. The paper presents a fuzzy expert system to get appropriate value of traverse speed. A CAPP system based on the fuzzy expert system for ASJ is also proposed in the paper. At last the application of the fuzzy expert system presents evidence of the usefulness of the system.

Key words: Traverse speed; Abrasive suspension jet; Fuzzy expert system; CAPP;

\section{INTRODUCTION}

Abrasive suspension jet (ASJ) is a new kind of AWJ (abrasive water jet). Shanghai University has put forward a new slurry and high pressure medium circle isolate device for ASJ (Patent No CN1597253). The device solves the problem of pollution between slurry and high pressure medium. And makes the slurry and high pressure medium recycle and reuse easily. It greatly reduces the user machining cost, and will help ASJ be more popular in mechanical machining [1].

There are two main stages in the cutting process. First stage is the preparative stage. The drawing will be transferred to G-code considering the special cutting process about abrasive suspension jet. The second is cutting stage. The numerical controller will control the movement of the nozzle according to the G-code for cutting. But the cutting process depends on a large number of parameters of an interconnected system. Many parameters show multi-causal behavior and cannot be optimized individually.

Please use the following format when citing this chapter:

Wang, Wenbin, Yu, Tao, Liu, Xiaojian, Liu, Tan, 2006, in International Federation for Information Processing (IFIP), Volume 207, Knowledge Enterprise: Intelligent Strategies In Product Design, Manufacturing, and Management, eds. K. Wang, Kovacs G., Wozny M., Fang M., (Boston: Springer), pp. 896-901. 
Furthermore. There is nonlinear relationship among these factors, and it is hard to give traditional mathematic formula. Though Hashish [2] has published an abrasive jet cutting model for predicting some process parameters for brittle materials. Kovacevic [3] used regress equation to predict relationship between traverse speed and cut depth. An error will always exist between the calculated and the actual results because of the simplification and assumption of the modeling. The parameter of traverse speed also encounters the same difficulty without exception, which is usually valued by experience. And the following problems often appear in the cutting stage:

First if the value of the traverse speed is too high, the jet will not cut through the material, it will spay and destroy the material;

Second if the value of the traverse speed is too low, the cost of cutting will be higher.

Third because the lag of jet, if the drawing is made up of complex curve, the unreasonable speed value will cause furrow in the rear of material during the jet turning the small corner, and lead to bad cutting quality.

So the paper presents an effective fuzzy decision method to get appropriate value of traverse speed. It includes five main sections. Section 2 discusses the fuzzy rule of constraints. Section 3 emphasizes on how to use fuzzy expert system to make the appropriate value of traverse speed automatically instead of the experience and expertise of the operator. Then the CAPP based on fuzzy expert system for ASJ has also been put forward in section 4 . Finally, deficiency about this system and conclusion are given in section 5 .

\section{FUZZY RULE OF CONSTRAINTS}

From above, some factors are imprecise and ambiguous such as the description about pressure, cutting quality and cost. These factors could not be described by the precise threshold, so it is necessary to fuzzify the traditional production rule. Thus the assumption has been made as follows :

- All rules should come up with multi-premise but single conclusion;

- The premise of rule admits the conjunction "AND" other than "OR". If the premise of one item include $\mathrm{K}$ times of "OR", the item of this rule must be considered as $\mathrm{K}+1$ items of single conclusion rule.

- The premise fuzzification: The fuzzy predication and fuzzy state measure word have been inducted in the premise. And the value of fuzzy membership degree also has been considered during fuzzy match. 
- Set the rule confidence CT $(0<\mathrm{CT}<1)$, so as to arrange the rule according to its $\mathrm{CT}$ value.

- The conclusion fuzzification.

\section{FUZZY EXPERT SYSTEM}

\subsection{Knowledge base}

The knowledge base has four components, as shown in Fig.2. The history database is used to store the appropriate traverse speed under different effect factors. So the history database will grow bigger and bigger with each time new factor has been concerned. The static database is used mainly to store the data of the traverse speed. The dynamic database is used to store all kinds of intermediate information acquired in the inferring process. The rule database represents the expert knowledge and theoretical knowledge abrasive suspension jet cutting. To set up the rule database the paper uses the method of fuzzy orthogonal test [4]. Thus various factors' effect to traverse speed can be evaluated more easily. Then the main domino effect has been used to calculate various membership degrees under different factors. So the rule's confidence can be arranged according the number of the fuzzy membership degree.

There are two kinds of representation of knowledge in the system.

Representation of facts-Predicate calculus is one of the methods used to represent knowledge. It is employed here to represent knowledge. Here the determination of the traverse speed is a combination of a set of facts. It can be represented by the conjunction of the predicates as follows:

Traverse speed $\wedge$ Material $\wedge$ Pressure $\wedge$ Nozzle parameters $\wedge \ldots$

Representation of reasoning knowledge - In the system production rules are used to represent reasoning knowledge. The general format is as follows:

IF (fact 1 exists)

(fact 2 exists)

...

(fact $\mathrm{n}$ exists)

Then (conclusion P)

\subsection{Inference system}

In developing the inference mechanism for expert systems, one of the inference strategies is to use both forward and backward chaining [5]. The 
system mainly uses forward chaining. The machining drawing gains from the CAD parts module or Image vectorization module. With the machining drawing has been split into series of lines and circles in sequence. Then the expert system will search history database first for the appropriate speed value, which matches the premise. If cannot find the answer in the history database, the exert system will infer the speed. And refer to the static database the appropriate value of traverse speed can be made. The appropriate value of traverse speed also has been recorded in the history database for using later. After pretreatment above the appropriate traverse speed of G-code can be made automatically. The whole workflow process is shown in Fig.1.

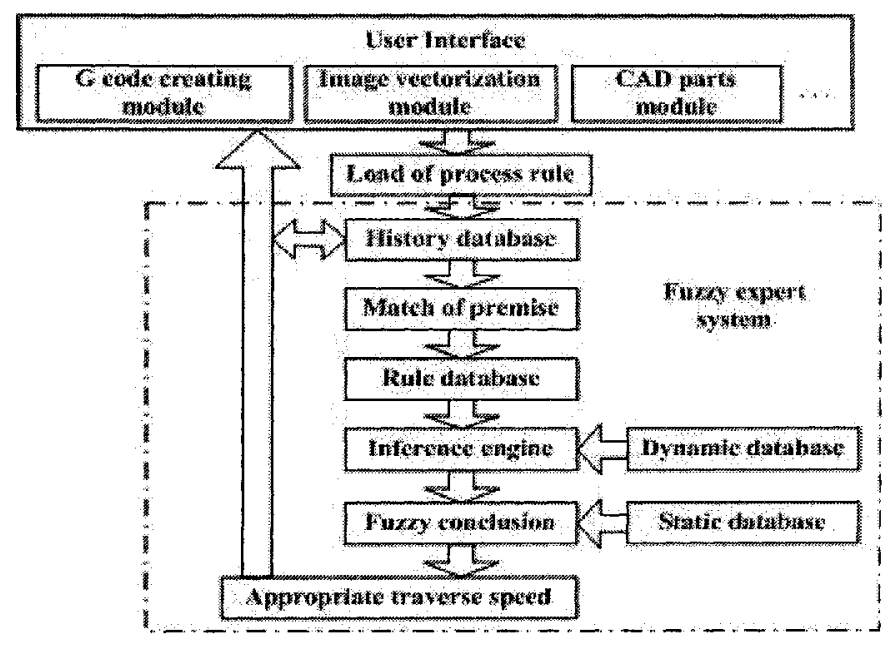

Figure 1. The workflow process of fuzzy expert system.

"Load of process rule" in the workflow means pick up each rule from the fuzzy knowledge base. Then use the conjunction "AND" to deal with multi premise rules.

\section{CAPP REALIZATION}

As is shown in Fig.2, First we get a AutoCAD design or image information, second we must specify known process parameters such as material parameters, nozzle parameters, slurry parameters, and etc, Then according to the fuzzy expert system the appropriate traverse speed is inferred. Third we specify working procedure such as original point, technical points, start point, end point, cutting path, cutting sequence and etc. thus the whole machining trace has been generated. Finally because some 
parts using a special technology need to be considered particularly, such as parts motion and rotation, cutter compensation and etc. The machining trace has been modified and the final trace code, G code, has been build by CAPP system.

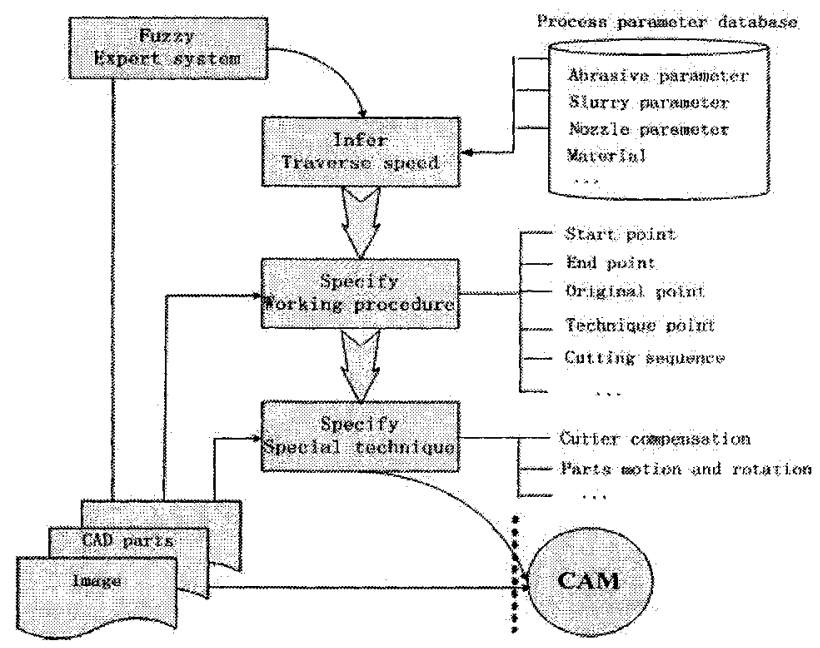

Figure 2. Flowchart of CAPP.

In this paragraph, we show how CAPP parts module makes man-machine interaction. In the left of Fig.3, drawing can be designed or modified in that area. And in the right, it is a CAPP plug in module, where points, lines, layers, dimensions, direction and some process parameters can be easily got. Also can interact with fuzzy expert system via this module to tell the process parameters such as the material, cutting pressure, the kind of abrasive and etc. Then fuzzy expert system generates appropriate traverse speed background. In the top right of Fig.3, it is a frame where G-code with the appropriate traverse speed has been made automatically.
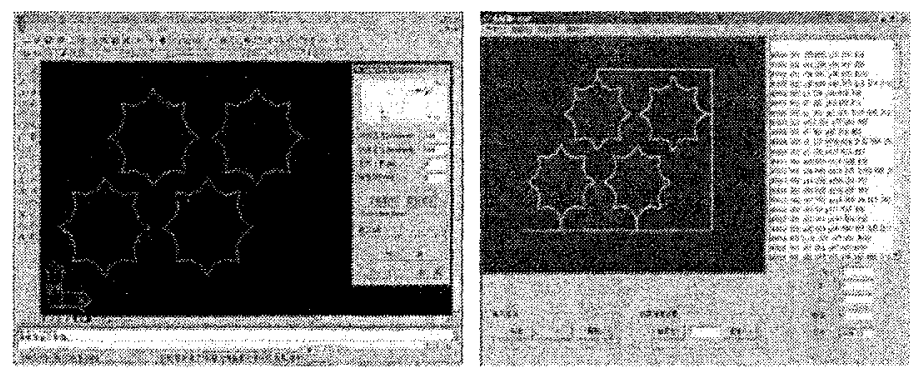

Figure 3. a). CAD parts module interface b) Realization interface 


\section{CONCLUSION}

The paper sets out from the application of the fuzzy expert system in the CAPP field for abrasive suspension jet. And emphasizes on how to use fuzzy expert system to make the appropriate value of traverse speed automatically. The running result show it can better simulate the expert thought in the field, realize the appropriate value of cutting speed and create G-code for working. It will be in favor of popularizing abrasive suspension jet in market.

Although this system has been designed and developed successfully, there is still no clearly estimate standard about cut quality of water-jet, so the expert system may be limited to some special field.

\section{REFERENCES}

1. Liu Xiaojian, Yu Tao, Liu Linsheng Wang Wenbin 'The study on abrasive suspension jet cutting technology", Lubrication Engineering, no. 169, pp. 31-33, 2005

2. Kovacevic,R.,et al., "Rehabilitation of the concrete pavements with abrasive waterjets", The $11^{\text {th }}$ International conference on jet cutting Technology, BRHA, st. Andrews Scotland, Sept, 8-10,1992;

3. Chakravarthy, P. Sitarama, Ramesh Babu, N. Source, "New approach for selection of optimal process parameters in Abrasive Water Jet cutting" Materials and Manufacturing Processes, v 14, n 4, Jul, 1999, p 581-600;

4. Honghua Su, Zhengjun Yao, "Fuzzy Analysis Method for Muti-Index Orthogonal Test", Journal of Nanjing University of Aeronautics \& Astronautics, Vol.36, No. 1, Feb, 2004;

5. Lawrence O. Hall, "Rule Chaining in Fuzzy Expert Systems", IEEE Transactions on Fuzzy Systems, Vol. 9, No. 6, Dec, 2001 